\title{
An Optimized Utilization of Battery Backup in MANET Using Modified Firefly Algorithm
}

\author{
Manoj Kumar ${ }^{1}$, Anshy Singh ${ }^{2}$ \\ ${ }^{1}$ Department of Computer Engineering and Application, GLA University, Mathura. \\ ${ }^{2}$ Department of Computer Engineering and Application, GLA university, Mathura. \\ manoj.kumar@gla.ac.in ${ }^{1}$, Anshy.singh@gla.ac.in²
}

Article History: Received: 10 November 2020; Revised: 12 January 2021; Accepted: 27 January 2021; Published online: 05 April 2021 Abstract: Temporary connection failures and route changes happen in a Mobile Ad Hoc Network (MANET). MANET
enjoys extensive variety of applications like in tactical networks, Sensor networks. Much battery backup is required while
tuning a node that is far from the sender node While compared to the node which is near in respect to sender, In this paper we
are proposing an approach of optimized utilization of battery backup in MANET Battery Backup is a main constraint in
mobile ad hoc networks Most of the battery is wasted in tuning to the networks repeatedly there by making the mobile node
vulnerable to Jail. This paper emphasize on proper utilization of battery backup by varying the signal strength according the
distance of the nodes. Modified FireFly Algorithm (MFFA) is greatly utilized in this research for boosting up of battery
backup. The cluster head should maintain a table for battery backup and decision of task distribution will be based on this
table.

Keywords: MANET Self Tuning DSDV, Signal Strength, Battery backup, optimization, Networks

\section{Introduction}

An ad-hoc mobile network made up of a group of peer mobile nodules that can correspond with each and every beyond the hold of a fixed infrastructure.

Although multi- hop packets are forwarded to each other, MANET is consist of a group of mobile wireless nodes which structure a network separately of any centralized administration [1-5]. The node interconnections are capable of constant and arbitrary transition. Nodes in the radio range of one another communicate straight through wireless connections, though those at a distance away utilize other nodes in the form of relays in a multihop routing manner [6-10]. A self-tuning system can optimize one's own internal running parameters to maximize or minimize an objective function's fulfillment. Ad hoc wireless networks are restricted in terms of power as nodes function with limited battery [11-13]. The path with a significant amount of the remaining battery is identified by the first flood, and the same time, the position of the route's neighboring nodes is determined from the radio power obtained. Through route link is divided into the power saving connection and tuned up by the second flood [14-19].

\section{Related Work}

Over the past couple of years, several MANETs have been proposed to discover protocols, however they generate substantial overhead traffic, and are therefore mainly suitable for small-sized MANETs having a restricted number of nodes. Many research works are projected in [20-23]. Mobile adhoc networking research has contributed to the expansion of several routing protocols and for continuous enhancement of the same. MANET routing protocols might be loosely split into constructive (e.g. DSDV [32], OLSR [33]), reactive (e.g. AODV [24]), hierarchal (e.g. ZRP [25]).

In any of these routing protocols there has been no consideration of improving the energy utilization of battery backup in nodes. In [26] some work has been carried out in direction of Tuning of the signal strength in MANET with human interaction. In our approach human interaction has been completely omitted, thereby making self tuning of MANETS possible Energy Efficient Utilization of Battery in Nodes [27-29]. A cluster head $(\mathrm{CH})$ will maintain one table consisting of node ids, hop count, battery backup of all nodes and distance of 1 hop neighbors in cluster. Initially, $\mathrm{CH}$ will send a request message for the distance and battery backup to all 1 hop neighbors [30-32]. On receiving the message from $\mathrm{CH}$ the 1hop neighbors will again send a request to its 1 hop neighbors requesting for their battery backup and this will propagate to end of the cluster [33]. Response message is sent back with battery backup and distance to cluster head [34-37].

\subsection{Example consider a network Diagram}

Figure 1 show the network diagram which is explained below. 


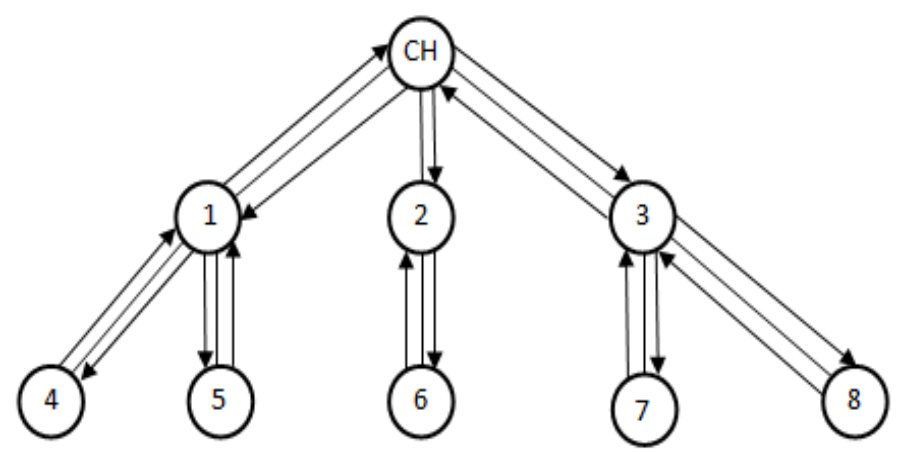

Figure 1. Network Diagram

Set 1: $\mathrm{CH}(\mathrm{CH})$

Set 2: $\mathrm{CH}$ 1hop neighbor $(1,2,3)$

Set3: 1hop neighbors to its neighbors $(4,5,6,7,8)$

Yellow arrows indicate request message from $\mathrm{CH}$ to 1 hop neighbors. REQ1 (BB, D)

Red arrows indicate the request message from nodes ( $\mathrm{CH} 1$ hop neighbor) to its 1hop neighbors. REQ2 (BB) Blue arrows indicate the response from node $(4,5,6,7,8)$ to $\mathrm{CH} 1$ hop neighbor as shown above.

\section{RESOURCE TABLE 1 (AT CH AFTER RECEIVING ALL THE RESPONSES)}

Table 1. At CH After Receiving All The Responses

\begin{tabular}{|l|l|l|l|}
\hline Node ID & Hop Count & Battery Backup & Distance \\
\hline 1 & 1 & 20 & 15 \\
\hline 2 & 1 & 30 & 10 \\
\hline 3 & 1 & 10 & 20 \\
\hline 4 & - & 20 & - \\
\hline 5 & - & 40 & - \\
\hline 6 & - & 20 & - \\
\hline
\end{tabular}

Depending on the distance entry in above table signal strength of 1 hop neighbors is self tuned by the $\mathrm{CH}$. For example for near node 1 the signal strength is less as compared to node 3.

Let average strength of the signal is 150 unit and by reducing 10 units of strength we save $5 \%$ of overall battery backup.

Following table shows the amount of saved battery backup.

\subsection{Formula}

In Table 2, Strength after self tuning, distance and saved power are shown. Saved battery backup $=($ Avg Strength-strength after self tuning) $* 5 \%$

Table 2. Strength after self tuning, distance and saved power

\begin{tabular}{|l|l|l|l|}
\hline Node ID & Strength after self tuning & Distance & Saved power \\
\hline 1 & 100 & 15 & $25 \%$ \\
\hline 2 & 70 & 10 & $40 \%$ \\
\hline 3 & 120 & 20 & $15 \%$ \\
\hline
\end{tabular}

Overall saved power (for node $1,2,3)=25+40+15=80 \%$

\section{Methodology}

In this research, MANT+MFFA algorithm is used to increase the proper utilization of battery backup by varying the signal strength according the distance of the nodes. Figure2 explains the complete architecture of methodology involved in this research. 


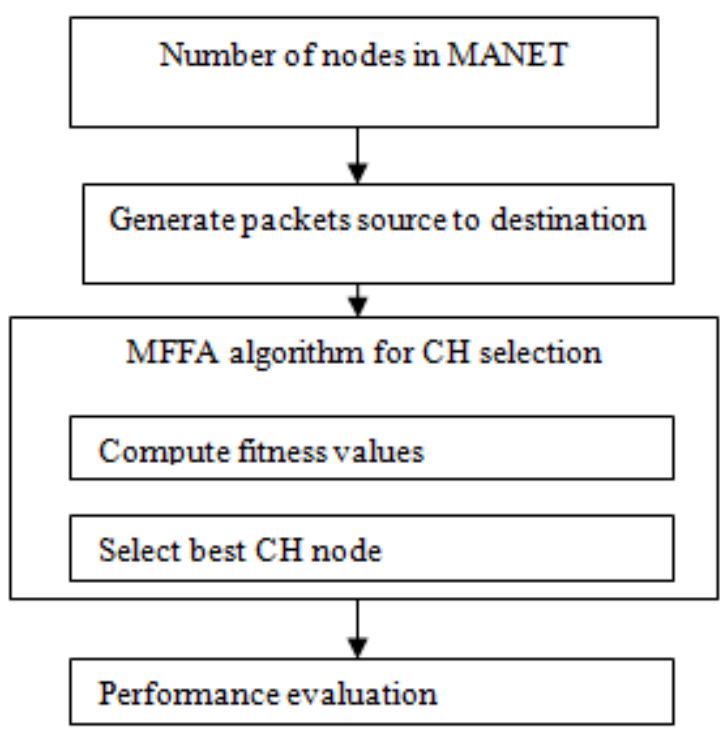

Figure 2. Overall architecture of the projected system

\subsection{CH selection using MFFA algorithm}

In this technical work, the $\mathrm{CH}$ node election is done with MFFA algorithm. The $\mathrm{CH}$ node election depends on the distance among the cluster and MANET node. Also it is based on the remaining energy present in the node in given network decreases the overhead of clustering process. It is used to reduce the load over $\mathrm{CH}$, avoiding reclustering and therefore lessen the power utilization within the cluster in large-scale.

Clustering is one among the fundamental procedure for broadening the system lifetime in MANET. It comprises of collection of the nodes into clusters and afterward choosing $\mathrm{CHs}$ for each group. CHs assemble the information from the nodes of the comparing group and advances the information collected to the fundamental node (Information focus). Appointment of $\mathrm{CH}$ is the cycle for choosing a specific node present inside the group to fill in as a head node. The $\mathrm{CH}$ inside the various leveled structure has a critical task to carry out in between group and intra-cluster correspondence. Hence, the $\mathrm{CH}$ plays to be the local coordinator for its part nodes and helps in the administration of the cluster individuals. A gateway node is fundamentally a node which implies an associating span between the between group and intra-cluster correspondence. Both the dispersed passages offer the way for between group correspondences. The standard nodes of the cluster fill in as the following prompt neighbors of the CHs. Figure 3 illustrate the general $\mathrm{CH}$ selection in MANET.

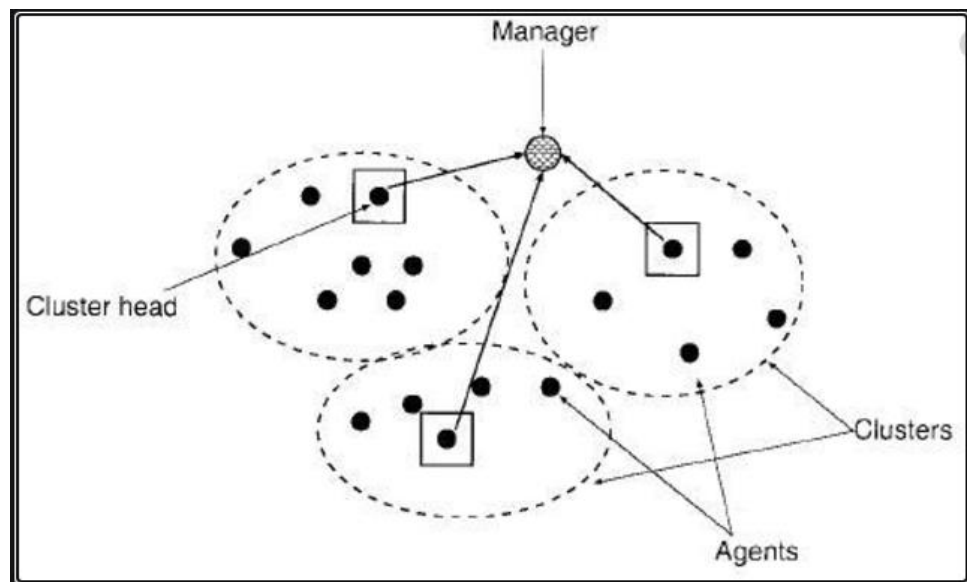

Figure 3. General $\mathrm{CH}$ selection in MANET

Firefly algorithm works on the concept of the firefly's illuminating lights. The intensity of the light supports a firefly's group shift to intense and more attractive positions that is plotted to produce best resolution over the seeking place. This mechanism normalizes few of the firefly features and can be shown as given below [38]: Structure of firefly algorithm is shown in Figure 4. 
i. $\quad$ Every firefly is attracted to a different irrespective of their sex.

ii. The brightness formed via the firefly is openly comparative to its attractiveness besides it is among two fireflies, the firefly along with more brightness attracts the one having lesser brightness. A firefly shifts arbitrarily when it is incapable to discover a brighter and nearest firefly.

iii. In the statistical form, the firefly brightness depends on objective function.

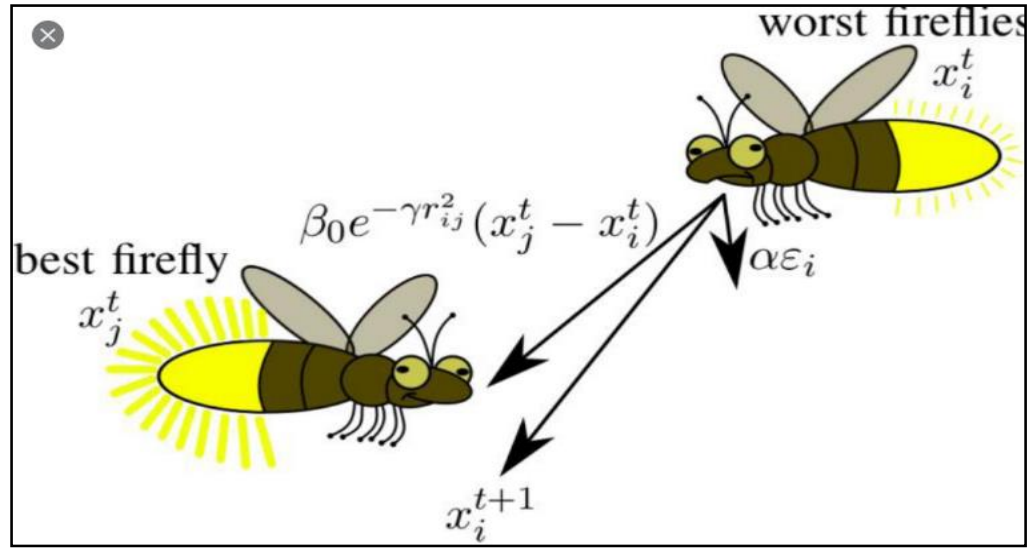

Figure 4. Structure of Firefly algorithm

Firefly algorithm is selected for its potential of giving best solutions for multi objective problems.

a) Attractiveness and Light Intensity at the source: The light intensity changes based on the inverse square law as below

$$
\mathrm{I}(\mathrm{r})=\frac{\mathrm{I}_{0}}{\mathrm{r}^{2}}
$$

Where $\mathrm{I}(\mathrm{r})$ is the light intensity at attractiveness $\mathrm{r}^{2}$

b) While the intermediate is provided, the light intensity is as given:

$\mathrm{I}(\mathrm{r})=\mathrm{I}_{0} \exp (-\gamma \mathrm{r})$

Where $I_{0}$ refers to the absorption coefficient of the medium

c) To prevent the singularity, the below Gaussian form of the approximation is used

$\mathrm{I}(\mathrm{r})=\mathrm{I}_{0} \exp \left(-\gamma \mathrm{r}^{2}\right)$

The firefly attractiveness is in proportion to light intensity observed through the neighboring fireflies. Hence firefly attractiveness $\beta$ is as given

$\beta=\beta_{0} \exp \left(-\gamma \mathrm{r}^{\mathrm{m}}\right)$

Where $\beta_{0}$ refers to the attractiveness at $r=0$.

Distance amid any two fireflies (nodes) $\mathrm{i}$ and $\mathrm{j}$ are positioned in addition the distance is computed as follows

$r_{i, j}=\sqrt{\sum_{k=1}^{d}\left(x_{i, k}-x_{j, k}\right)^{2}}$

Where $\mathrm{x}_{\mathrm{i}, \mathrm{k}}$ indicates $\mathrm{kth}$ factor of spatial match $\mathrm{x}_{\mathrm{i}}$ of kth firefly, $\mathrm{d}$ stands for amount of dimensions.

In this research, a new fitness function assumes energy consumption, and delay which is given by

$f(x)=\frac{\left(\mathrm{m}_{\mathrm{d} / \mathrm{m}_{\mathrm{t}}}\right) \times\left({ }^{\mathrm{P}_{\mathrm{i}}^{\mathrm{r}} / \mathrm{P}_{\text {init }}}\right)}{\exp ^{-{ }^{\mathrm{e}} \mathrm{D} / \mathrm{e} / \mathrm{M}}}$

wherem $m_{d}$ is the number of dropped messages. $m_{t}$ refers to the total number of messages transmitted.

$\mathrm{P}_{\mathrm{i}}^{\mathrm{r}}$ indicates power remaining in node $\mathrm{i}$.

$P_{\text {init }}$ refers to the initial power.

$\mathrm{e}_{\mathrm{D}}$ indicates the end to end delay and $\mathrm{e}_{\mathrm{M}}$ refers to the maximum permissible delay.

$\mathrm{x}_{\mathrm{i}}=\mathrm{x}_{\mathrm{i}}+\beta_{0} \mathrm{e}^{-\gamma \mathrm{r}^{2}}\left(\mathrm{x}_{\mathrm{j}}-\mathrm{x}_{\mathrm{i}}\right)+\alpha\left(\right.$ rand $\left.-\frac{1}{2}\right)$

Where $x_{i}$ and $x_{j}$ is distance between two firefly nodes 
The cluster creation and $\mathrm{CH}$ election in MFFA are provided in algorithm

\section{Algorithm 1: MFFA algorithm}

1. Objective function $(x), x=(x 1, \ldots)$,

2. Produce initial population of fireflies $x i(i=1,2, \ldots, n)$

3. Light intensity $I i$ at $x i$ is found via $f(x i)$

4. Describe light absorption coefficient $\gamma$

5. while $(t<$ MaxGeneration)

6. for $i=1: n$ all $n$ fireflies

7. for $j=1: i$ all $n$ fireflies

8. if $(I j>I i)$, Transfer firefly $i$ towards $j$ in $d$-dimension;

9. end if

10. Attractiveness changes along with distance $r$ according to $\exp [-\gamma r]$

11. Compute fitness function using (6) and (7)

12. Compute objective model

13. Estimate new solutions and update light intensity using (2)

14. end for $j$

15. end for $i$

16. Sort the fireflies and get current best

17. end while

18. A firefly i shifts to a more attractive

In this scenario, MFFA algorithm is used to produce optimal solutions by improving the energy and delay metrics. The binary values are utilized to symbolize the nodes in every solution. In the firefly algorithm, the fireflies are ranked and the optimal fireflies are chosen by best fitness values. The chosen fireflies replicate between themselves through crossover and mutation. The novel best solutions are appended to the firefly group and the subsequent iteration of the firefly is then proceeded. Hence, in this technical work, $\mathrm{CH}$ node is elected with the help of MFFA algorithm in accordance to the reduced energy depletion, distance and maximum packet delivery and lower number of hop count nodes. It is focused to select the optimal $\mathrm{CH}$ through multi objective function for the resulted clusters.

\section{Simulation result}

This section discusses the performance evaluation and comparison between the discussed MFFA technique and available techniques such as OLSR and AODV. The experiments are performed by greatly utilizing NS-2 simulator. Also, the system is validated by comparing existing and suggested techniques pertaining to metrics such as end to end delay, throughput, energy consumption, as well as network lifetime. The simulation settings are given in Table 3 .

Table 3. Simulation Parameters

\begin{tabular}{|l|l|}
\hline Parameter & values \\
\hline No. of Noes & 100 \\
\hline Area Size & 1100 X $1100 \mathrm{~m}$ \\
\hline Mac & 802.11 \\
\hline Radio Range & $250 \mathrm{~m}$ \\
\hline Simulation Time & $60 \mathrm{sec}$ \\
\hline Packet Size & $80 \mathrm{bytes}$ \\
\hline
\end{tabular}

\subsection{Performance Evaluation}

\section{End-to-end delay}

The standard time taken for packet transmission from source to destination nodes over the system is called as end to end delay 


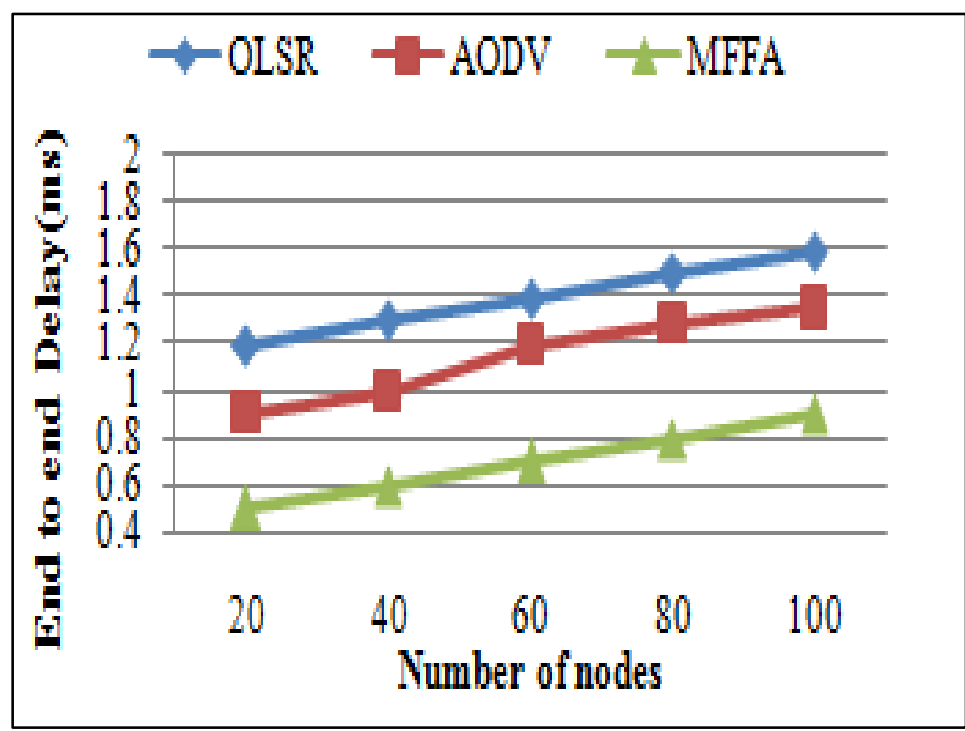

Figure 5. Comparison of End-to-end delay

The end to end delay performance is compared between proposed MFFA and prevailing OLSR and AODV methods which is revealed in Figure 5.Graph illustrating the performance is obtained by taking number of nodes in $\mathrm{x}$ axis and end to end delay in y axis. The nodes vary amid 20 and 100 and end to end delay (ms) is plotted for these nodes. It is thereby precise that the discussed MFFA algorithm achieves less end to end delay than available OLSR and AODV algorithms.

\section{Throughput}

The speed of meaningful information transmission across system or links of communication is called as throughput. It is estimated in bits per second (bit/s or bps). It is furthermore indicated through units of packets processed in a specified period.

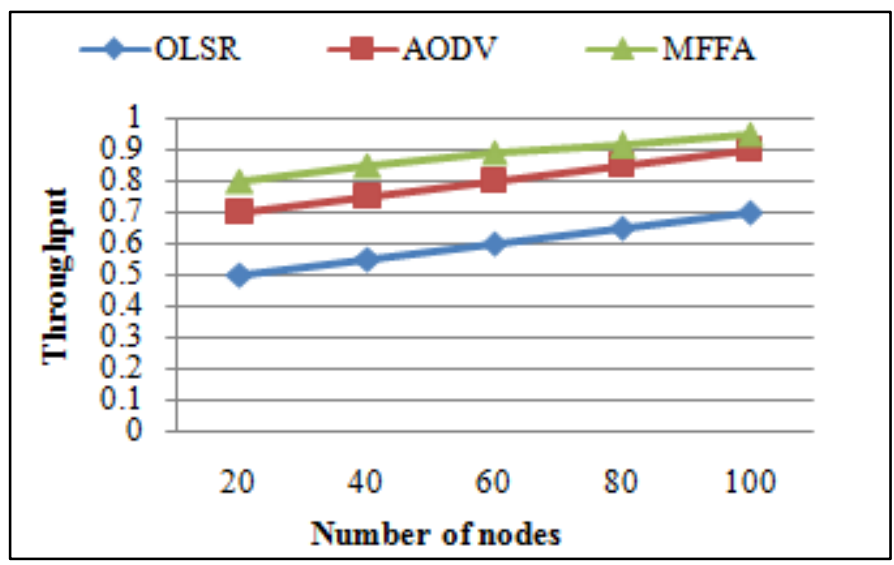

Figure 6. Throughput comparison

Figure 6 illustrates the comparison between throughput performance of the discussed MFFA and available OLSR and AODV approaches. The number of nodes are plotted along $\mathrm{x}$ axis and throughput is plotted along $\mathrm{y}$ axis. It is apparent from the graph that suggested MFFA algorithm produces considerably improved throughput compared to available techniques of OLSR and AODV.

\section{Energy consumption}

It refers to the standard power needed for the send and receive functions of a packet to a node present in system in the course of a time slot specified. 


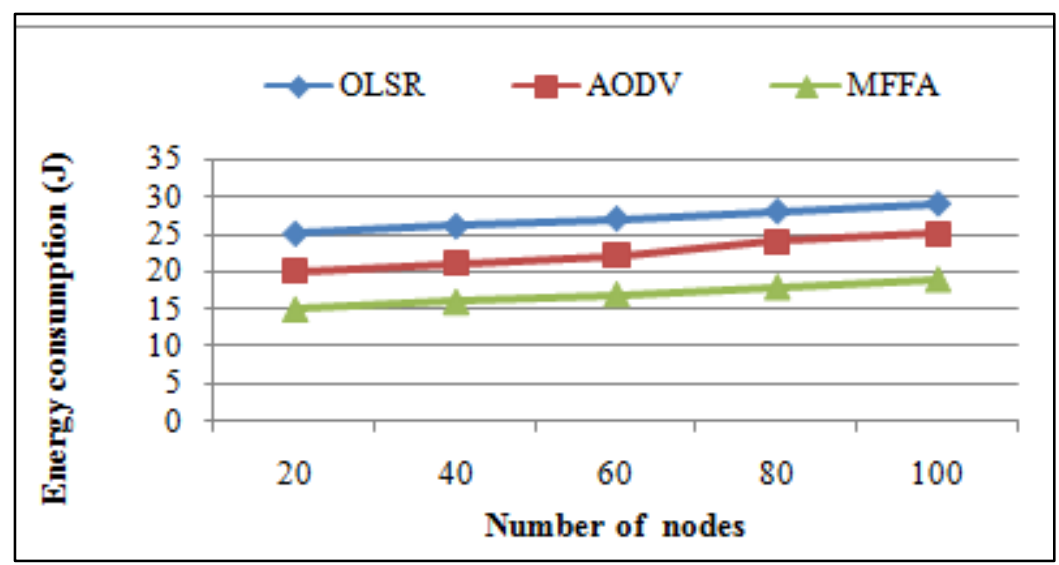

Figure 7. Energy consumption comparison

Figure 7 illustrates the performance comparison pertaining to energy consumption for suggested MFFA in addition to available OLSR and AODV approaches. The number of nodes are plotted along $\mathrm{x}$ axis and the energy consumption is plotted along y axis. It is apparent from the graph that suggested MFFA scheme produces enhanced energy consumption compared to available techniques.

\section{Network lifetime}

The system is known to be good if the proposed technique achieves improved network lifespan.

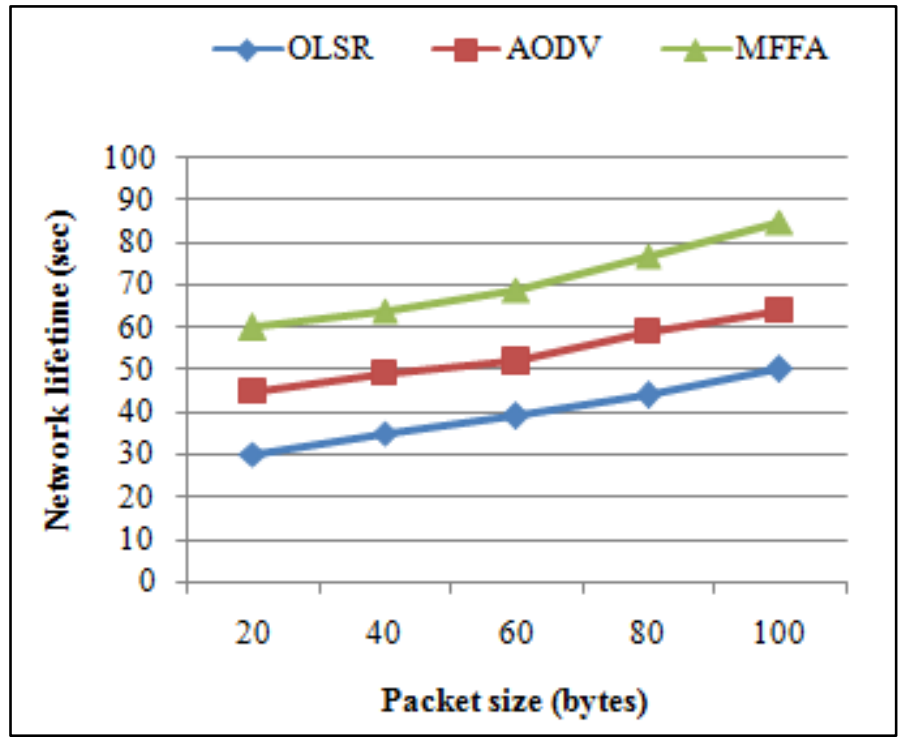

Figure 8. Network lifetime

Figure 8 illustrates network lifespan for the packet size provided. The packet size is strategized along the $\mathrm{x}$ axis and the network lifespan is taken along y axis. The present system yields much lesser network performance in comparison with the proposed system achieving better performance. The discussed system thereby improves network lifespan through preventing repetitive exploitation of nodes if there is an increase in packet size. It is revealed that MFFA algorithm produces superior network lifespan when compared to other prevailing OLSR and AODV methods.

\section{Conclusion and Future Work}

A methodology is suggested for solving a major problem in MANET by saving the battery backup of CH by self tuning for nodes at different distances, this method can also be applied for other nodes in the network. As shown in above calculation overall energy conservation is as high as $80 \%$. For future work, resource table can be optimized. 


\section{References}

1. Sailhan, F. Issarny, V. Pervasive Computing and Communications, 2005. PerCom 2005. Third IEEE International Conference on On page(s): 235-244 ISBN: 0_7695-2299-8 121.Publication Date: 8-12 March 2005.

2. P. E. Engelstad , Y. Zheng, Evaluation of Service Discovery Architectures for Mobile Ad Hoc Networks, Proceedings of the Second Annual Conference on Wireless On-demand Network Systems and Services, p.2-15, January 19-21, 2005

3. Francoise Sailhan, Valerie Issarny, Scalable Service Discovery for MANET, Proceedings of the Third IEEE nternational Conference on Pervasive Computing and Communications, p.235-244, March 08-12, 2005[doi>10.1109/PERCOM 2005.36]

4. Coulouris , Jean Dollimore, Tim Kindberg, Distributed Systems: Concepts and Design (4th Edition) (International Computer Science), Addison-Wesley Longman Publishing Co., Inc., Boston, MA, 2005

5. E. Guttman, C. Perkins, J. Veizades, M. Day, Service Location Protocol, Version 2, RFC Editor, 1999

6. K Mausam, K Sharma, G Bharadwaj, RP Singh, "Multi-objective optimization design of diesinking electric discharge machine (EDM) machining parameter for CNT-reinforced carbon fibrenanocomposite using grey relational analysis, Journal of the Brazilian Society of Mechanical Sciences and Engineering" $41 \ldots$

7. MK Shukla, K Sharma, Improvement in mechanical and thermal properties of epoxy hybrid composites by functionalized graphene and carbon-nanotubes, Materials Research Express 6 (12), 125323

8. K Kumar, K Sharma, S Verma, N Upadhyay, Experimental Investigation of Graphene-Paraffin Wax Nanocomposites for Thermal Energy Storage, Materials Today: Proceedings 18, 5158-5163

9. Goyal, M., Shape, size and phonon scattering effect on the thermal conductivity of nanostructures. Pramana, 91(6): p. 87, 2018.

10. Goyal, M. and B. Gupta, Study of shape, size and temperature-dependent elastic properties of nanomaterials. Modern Physics Letters B, 33(26): p. 1950310.2019.

11. Goyal, M. and B. Gupta, Analysis of shape, size and structure dependent thermodynamic properties of nanowires. High Temperatures--High Pressures, 2019. 48.

12. Goyal, M. and M. Singh, Size and shape dependence of optical properties of nanostructures. Applied Physics A, 126(3): p. 1-8, 2020.

13. Sun Microsystems. Jini network technology. http://www.jini.org, 1999.

14. S. Nesargi and R. Prakash. Issues pertaining to service discovery in mobile ad hoc networks. ACM Workshop on Principles of Mobile Computing, 2001

15. R. Beraldi, L. Querzoni, R. Baldoni. A hint-based probabilistic protocol for unicast communications in manets. Elsevier Ad Hoc Networs, 2005.

16. SantashilPalChaudhuri, Jean-Yves Le Boudec, Milan Vojnovic, Perfect Simulations for Random Trip Mobility Models, Proceedings of the 38th annual Symposium on Simulation, p.72-79, April 04-06, 2005[doi>10.1109/PERCOM 2005.33]

17. U.C. Kozat and L. Tallius. Service discovery in mobile ad hoc networks: an overall perspective on architectural choices and network layer support issues. Ad Hoc Networks, (2)2004.

18. V. Lenders, M. May, B. Plattner. Service discovery in mobile ad hoc networks: A field theoretic approach. Elsevier Pervasive and Mobile Computing, 2005.

19. C. Perkins and P. Bhagwat. Highly Dynamic Destination-Sequences Distance Vector Routing (DSDV) for Mobile Computers. In Proceedings of ACM SIGCOMM 1994, London, UK, 1994.

20. T. Clausen and P. Jacquet. Optimized Link State Routing Protocol (OLSR). RFC 2326, October 2003

21. A Kumar, K Sharma, AR Dixit A review of the mechanical and thermal properties of graphene and its hybrid polymer nanocomposites for structural applications, Journal of materials science 54 (8), 5992-6026.

22. K Sharma, M Shukla, Three-phase carbon fiber amine functionalized carbon nanotubes epoxy composite: processing, characterisation, and multiscale modeling, Journal of Nanomaterials 2014

23. K Sharma, KS Kaushalyayan, M Shukla, Pull-out simulations of interfacial properties of amine functionalized multi-walled carbon nanotube epoxy composites, Computational Materials Science $99,232-241$ 
24. A Yadav, A Kumar, PK Singh, K Sharma, Glass transition temperature of functionalized graphene epoxy composites using molecular dynamics simulation, Integrated Ferroelectrics 186 (1), 106114

25. PK Singh, K Sharma, A Kumar, M Shukla, Effects of functionalization on the mechanical properties of multiwalled carbon nanotubes: A molecular dynamics approach, Journal of Composite Materials 51 (5), 671-680

26. PK Singh, K Sharma, Mechanical and Viscoelastic Properties of In-situ Amine Functionalized Multiple Layer Grpahene/epoxy Nanocomposites, Current Nanoscience 14 (3), 252-262

27. Singh PK, \& Sharma K, Molecular Dynamics Simulation of Glass Transition Behaviour of Polymer based Nanocomposites, Journal of Scientific \& Industrial Research, 77 (10) 592-595. (2018).

28. A Kumar, K Sharma, AR Dixit, Carbon nanotube-and graphene-reinforced multiphase polymeric composites: review on their properties and applications, Journal of Materials Science, 1-43

29. MK Shukla, K Sharma, Effect of carbon nanofillers on the mechanical and interfacial properties of epoxy based nanocomposites: A review, Polymer Science, Series A 61 (4), 439-460

30. A Kumar, K Sharma, AR Dixit, A review on the mechanical and thermal properties of graphene and graphene-based polymer nanocomposites: understanding of modelling and MD simulation, Molecular Simulation 46 (2), 136-154

31. C.E. Perkins, E.M. Belding-Royer, and S.R. Das. Ad hoc On-Demand Distance Vector (AODV) Routing. Experimental RFC 3561, July 2003.

32. Z.J. Haas and M.R. Pearlman. The Performance of Query Control Schemes for the Zone Routing Protocol. In Proceedings of ACM SIGCOMM 1998, Vancouver, 1998.

33. Mobile Ad Hoc Networking. Edited by Basagni, Conti, Giordano, and Stojmenovic. Institute of Electrical and Electronics Engineers, Inc.447 ISBN 0-471-37313-3 @ 2004

34. Sharma, Ashish, Anant Ram, and Archit Bansal. "Feature Extraction Mining for Student Performance Analysis." Proceedings of ICETIT 2019. Springer, Cham, 785-797,2020.

35. Sharma, Ashish, Ashish Sharma, and Anand Singh Jalal. "Distance-based facility location problem for fuzzy demand with simultaneous opening of two facilities." International Journal of Computing Science and Mathematics 9.6 590-601(2018).

36. Ram, Anant, et al. "A density based algorithm for discovering density varied clusters in large spatial databases." International Journal of Computer Applications 3.6 1-4(2010).

37. Mishra, Ayushi, et al. "A robust approach for palmprint biometric recognition." International Journal of Biometrics 11.4 389-408 (2019).

38. Prakash, SKLV Sai, and Kondapalli S. Rami Reddy. "Firefly inspired energy aware cluster based tree formation in WSN." 2014 2nd International Conference on Information and Communication Technology (ICoICT). IEEE, 2014. 